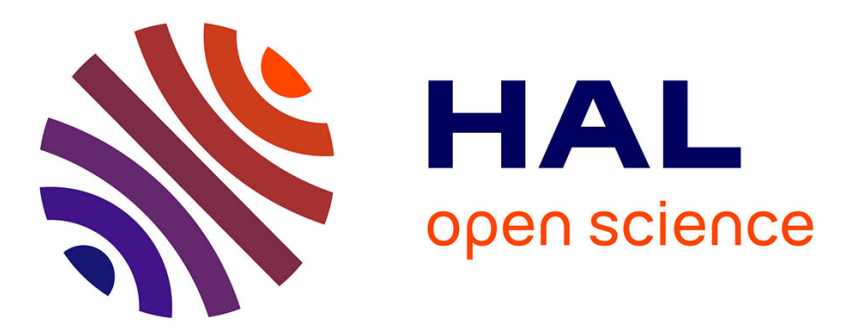

\title{
L'impact de l'agriculture sur les incendies de forêt et leur propagation dans les régions méditerranéennes françaises
}

Francesco Guerra, Claude Napoleone, Jean Christophe Paoli, Michel Moulery

\section{- To cite this version:}

Francesco Guerra, Claude Napoleone, Jean Christophe Paoli, Michel Moulery. L'impact de l'agriculture sur les incendies de forêt et leur propagation dans les régions méditerranéennes françaises. Cahiers de la Méditerranée, 2021, 102, pp.145-161. 10.4000/cdlm.14660 hal-03472036

\section{HAL Id: hal-03472036 https://hal.inrae.fr/hal-03472036}

Submitted on 9 Dec 2021

HAL is a multi-disciplinary open access archive for the deposit and dissemination of scientific research documents, whether they are published or not. The documents may come from teaching and research institutions in France or abroad, or from public or private research centers.
L'archive ouverte pluridisciplinaire HAL, est destinée au dépôt et à la diffusion de documents scientifiques de niveau recherche, publiés ou non, émanant des établissements d'enseignement et de recherche français ou étrangers, des laboratoires publics ou privés. 


\section{Cahiers de la Méditerranée}

102 | 2021

Le foncier et la marginalité en Méditerranée

\section{L'impact de l'agriculture sur les incendies de forêt et leur propagation dans les régions méditerranéennes françaises}

The impact of agriculture on wildfires and their spread in the French Mediterranean regions

Francesco Guerra, Claude Napoléone, Jean Christophe Paoli et Michel Moulery

\section{OpenEdition \\ Journals}

Édition électronique

URL : https://journals.openedition.org/cdlm/14660

DOI : $10.4000 / \mathrm{cdlm} .14660$

ISSN : 1773-0201

Éditeur

Centre de la Méditerranée moderne et contemporaine

Édition imprimée

Date de publication : 15 juin 2021

Pagination : 145-161

ISSN : 0395-9317

Ce document vous est offert par INRAE Institut National de Recherche pour l'Agriculture,

I'Alimentation et l'Environnement

\section{INRAC}

Référence électronique

Francesco Guerra, Claude Napoléone, Jean Christophe Paoli et Michel Moulery, « L'impact de l'agriculture sur les incendies de forêt et leur propagation dans les régions méditerranéennes françaises », Cahiers de la Méditerranée [En ligne], 102 | 2021, mis en ligne le 01 décembre 2021, consulté le 08 décembre 2021. URL : http://journals.openedition.org/cdlm/14660 ; DOI : https:// doi.org/10.4000/cdlm.14660

Ce document a été généré automatiquement le 8 décembre 2021.

(C) Tous droits réservés 


\title{
L'impact de l'agriculture sur les incendies de forêt et leur propagation dans les régions méditerranéennes françaises
}

\author{
The impact of agriculture on wildfires and their spread in the French \\ Mediterranean regions
}

Francesco Guerra, Claude Napoléone, Jean Christophe Paoli et Michel Moulery

Cette recherche a été financée par le programme PROVIDE (PROVIding smart DElivery of public goods by EU agriculture and forestry - H2020-ISIB-2014-2, Topic : ISIB-01-2014).

Les auteurs remercient Jean-Frédéric Biscay (commandant du Cesir de Valabre - Gardanne), Eric Rigolot (chercheur, URFM - INRA PACA) et Michel Costa (chef de la commission de prévention des feux de forêt de Corse du sud) pour leurs commentaires et suggestions.

1 Les incendies de forêt sont un enjeu important dans les régions méditerranéennes ${ }^{1}$ et le changement climatique est susceptible d'en accroitre le risque ${ }^{2}$. Les conséquences vont de la destruction de l'environnement ${ }^{3}$ à la perte de biens privés ${ }^{4}$. En outre, les nombreuses actions gouvernementales visant à prévenir et à combattre les feux de forêt sont coûteuses, tandis que la régénération naturelle des zones brûlées est lente ${ }^{5}$. Dans ce contexte, il convient de s'interroger sur l'effet des différents patrons paysagers sur l'occurrence et la propagation des incendies de forêt, en ce sens que l'estimation et la localisation de cette relation permettent de focaliser l'action de prévention ou de lutte sur les espaces où la probabilité d'occurrence est importante. Dans cette perspective, un regard particulier a été accordé aux zones agricoles, principalement du fait que leur relation au risque d'incendie est relativement peu documentée alors qu'elle est non homogène, c'est-à-dire imprévisible à partir d'une simple cartographie des usages: le concours des productions agricoles à la présence de biomasse combustible pendant les mois d'été n'est pas équivalent entre les cultures maraichères, 
les cultures irriguées, les vignobles non enherbés ou les céréales. En outre, les espaces agricoles situées à proximité de zones sujettes aux incendies constituent une source d'occurrence de feux (par le contact entre activité humaine et biomasse combustible ${ }^{6}$ ) mais, en même temps, ils peuvent les retarder par la facilitation des interventions de lutte contre les incendies ${ }^{7}$.

2 Pour évaluer le lien entre les différents types d'espaces agricoles et le risque d'incendie, nous avons conduit une analyse statistique de l'occurrence et de la propagation des incendies à partir du recensement des feux passés et des données géomatiques sur les usages des sols, sur une période de huit ans et dans la zone méditerranéenne française. Tout d'abord, nous avons caractérisé l'impact de l'utilisation des terres agricoles sur le nombre d'occurrence des incendies, grâce à un modèle binomial négatif dit à excès de zéros (zero inflated models). Dans un deuxième temps, nous avons utilisé un modèle Logit pour estimer dans quelle mesure l'agriculture empêche un feu allumé d'atteindre des étendues importantes. Les résultats de l'analyse nous permettent de dresser le profil des municipalités qui sont plus exposées au risque d'incendie de forêt eu égard à la présence de certains types de cultures ou d'activités agricoles.

3 Le texte est structuré en trois sections. La première est une revue de la littérature récente sur l'analyse spatiale des modèles d'incendies de forêt, en mettant l'accent sur la zone méditerranéenne. Nous montrons que, bien que la relation entre le risque d'incendie de forêt et les différents types de couverture terrestre ait été traitée, l'aspect agricole reste peu étudié. Dans la deuxième section, nos données sont décrites et notre méthodologie expliquée. La troisième section présente nos résultats et ouvre une discussion au sein de laquelle nous interrogeons les limites des approches disciplinaires mobilisées, l'hétérogénéité du terrain étudié ainsi que l'échelle d'observation.

\section{État de l'art}

4 La région méditerranéenne est la partie de l'Europe la plus touchée par les feux de forêt, même si sa part $(55 \%)$ suit une tendance à la baisse au cours des dernières décennies ${ }^{8}$. De fait, la sensibilisation croissante à ce risque a suscité de nombreuses études sur les facteurs qui influencent l'apparition et le déroulement des incendies de forêt, dans le but d'en améliorer la prévention et les stratégies de gestion des zones les plus sensibles. De nombreuses ont porté sur la péninsule ibérique ${ }^{9}$, la zone européenne qui a historiquement le plus connu d'incendies de forêt et de superficies brûlées ${ }^{10}$, alors que relativement moins d'études ont porté sur le sud de la France ${ }^{11}$, l'Italie ${ }^{12}$ et la Grèce ${ }^{13}$.

5 La plupart des incendies de forêt en zone méditerranéenne se produisent entre juillet et août, lorsque des températures élevées et une faible humidité, tant dans l'air que dans la végétation couvrant le sol, créent les conditions nécessaires à l'allumage et la propagation $\mathrm{du} \mathrm{feu}^{14}$. Les valeurs climatiques ${ }^{15}$ et la fréquence des pluies estivales ${ }^{16} \mathrm{en}$ sont un prédicteur d'occurrence. Peu d'études concluent toutefois que la dynamique météorologique est la principale cause des incendies de forêt ${ }^{17}$ : il y a un quasiconsensus pour mettre en exergue l'impact de l'utilisation et la couverture des sols ${ }^{18}$ sur l'occurrence et l'étendue des incendies, en se focalisant sur les causes anthropiques ${ }^{19}$. Formellement, la relation entre activité anthropique et risque d'incendie transite par différents vecteurs : économiques lorsque les zones arbustives sont plus sujettes aux risques d'incendie que les forêts du fait de leur moindre valeur ${ }^{20}$, 
géographiques quand la plupart des incendies se produisent à proximité des routes et des zones urbaines ${ }^{21}$, ou encore topographiques lorsque l'altitude ou la rugosité modifient le niveau de risque ${ }^{22}$ (l'exemple caractéristique est qu'au-dessus de 2000 mètres, la probabilité d'un incendie est considérablement plus faible ${ }^{23}$ ). Dès lors que les analyses sont menées à l'échelle régionale ou départementale, des variables socioéconomiques sont également convoquées, telles que le chômage ${ }^{24}$, le taux de pauvretée ${ }^{5}$ ou l'emploi par secteur ${ }^{26}$.

6 En ce qui concerne la relation entre l'agriculture et le risque d'incendie, la littérature actuelle est peu abondante et ne peut être interprétée de manière univoque. Certaines études mettent en évidence la corrélation positive entre l'utilisation des terres agricoles et le nombre d'incendies ${ }^{27}$, tandis que d'autres décrivent les zones agricoles comme moins sujettes à des incendies de grande étendue ${ }^{28}$; les résultats diffèrent en fonction de l'ampleur et du type d'incendie considéré. Si l'on prend en compte tous les incendies, y compris ceux qui n'affectent pas les espaces naturels, les zones agricoles semblent plus sujettes aux incendies en raison des activités humaines. Lorsque les incendies de plus grande ampleur sont pris en compte seuls, les zones agricoles y semblent moins sujettes que les zones arbustives et les forêts. L'effet de l'agriculture sur le risque d'incendie n'est donc pas monotone et nous pouvons supposer que cette relation dépend de relations complexes entre les types d'agriculture et les espaces naturels environnants.

7 Plusieurs propositions permettent de modéliser dans un cadre unifié le rôle des activités humaines dans les régimes d'occurrence et de propagation des incendies, en utilisant des approches statistiques spatiales ou temporelles ${ }^{29}$. L'analyse spatiale porte principalement sur la distribution des feux de forêt en relation avec les variables topographiques et d'utilisation $\mathrm{du} \mathrm{sol}^{30}$. Les analyses temporelles reposent généralement sur des variables climatiques et d'humidité des combustibles et visent à prédire l'occurrence ou la propagation des incendies au sein d'unités spatiales présentant des caractéristiques homogènes ${ }^{31}$. Ces analyses n'incluent que très peu les facteurs météorologiques ${ }^{32}$, même si dans certains cas, les variables climatiques annuelles moyennes telles que les précipitations ou la température s'avèrent significatives ${ }^{33}$. Il est à noter que même si les analyses spatiales sont généralement menées sur plusieurs années, leur dimension temporelle est relativement pauvre, principalement du fait de la non-homogénéité des données au fil du temps. Toutefois, Prestemon et al. ${ }^{34}$ ont pu constater dans les forêts de Floride, un risque d'inflammation plus faible pour les espaces où un incendie s'était déclaré au cours des sept années précédentes. A contrario, Díaz-Delgado et al. ${ }^{35}$ montrent que dans la région méditerranéenne, la fréquence des incendies tend à être plus élevée dans les zones précédemment brûlées après une période de quelques années, du fait de la vigueur de la régénération naturelle de la végétation arbustive.

8 Au niveau méthodologique, différents modèles ont été utilisés pour expliquer la variabilité de l'activité des feux de forêt entre les unités d'analyse temporelles et spatiales. Il s'agit notamment de modèles d'événements qui rendent compte de toutes les occurrences de feu, quelle que soit la zone brûlée, de modèles d'extension ou de dommages qui se concentrent sur les facteurs influençant la propagation ou l'intensité d'un incendie, ou encore de combinaisons des deux types ${ }^{36}$. Les modèles d'événements les plus courants analysent l'occurrence d'un feu sous forme de comptage et recourent à différents types de modélisation. Ils étudient, par exemple, l'occurrence des feux de 
forêt comme un événement binaire et sont capables d'incorporer des relations spatialement et temporellement dépendantes ou autorégressives. Les régressions logistiques ont ainsi été largement utilisées pour prévoir les jours d'incendie à partir des informations quotidiennes sur les conditions météorologiques ${ }^{37}$, pour décrire les schémas spatiaux d'allumage ${ }^{38}$ ou pour relier le nombre d'allumage sur une période et un espace définis à un ensemble de covariables différentiées ${ }^{39}$. D'autres modèles prennent en compte la taille de l'incendie plutôt que sa fréquence et relient l'étendue globale ou ponctuelle d'un incendie à un ensemble de variables explicatives supposées influencer leur propagation ${ }^{40}$. Dans cette perspective, des outils économétriques permettent d'estimer indépendamment deux éléments conjoints (comme l'occurrence et l'étendue); les modèles en deux étapes de Heckman et Tobit en sont un exemple ${ }^{41}$.

\section{Matériaux et méthode}

\section{Une aire d'étude hétérogène}

9 Le climat méditerranéen est caractérisé par des étés chauds et secs, et des hivers humides et doux. Les précipitations moyennes pendant les trois mois d'été varient de 31 à $54 \mathrm{~mm}$, tandis que les températures moyennes varient entre 19 et 22 degrés. Ayant retenu les 2731 communes des trois régions françaises bordant la Méditerranée (Provence/Alpes/Côtes d'Azur (PACA), Languedoc-Roussillon (LR ${ }^{42}$ et Corse (Corse)), notre zone d'étude couvre $67456 \mathrm{~km}^{2}$ (Figure 1). Les espaces naturels sont dominés par une végétation méditerranéenne composée de buissons et d'arbustes, appelée maquis ou garrigue selon la formation végétale, qui correspond soit à un état issu de la dégradation des forêts après incendie, soit à l'abandon agricole. Viticulture, oléiculture et différents types de céréaliculture sont les activités agricoles dominantes, tandis que l'élevage est principalement pratiqué dans les zones montagneuses. Dans ces trois régions, la plaine côtière est encadrée par des montagnes (plus de la moitié des communes sont classées en zone montagne - sources: ANEM). Les incendies y sont fréquents et touchent à la fois les forêts et les zones arbustives, qui représentent respectivement $34 \%$ et $22 \%$ de leur superficie totale.

Cette zone se caractérise en outre par une grande hétérogénéité, notamment en termes d'anthropisation. On y trouve deux départements très peu peuplés (les Alpes de Haute Provence - 24 hab. $/ \mathrm{km}^{2}$ - et la Lozère - $15 \mathrm{hab} . / \mathrm{km}^{2}$ ) et d'autres beaucoup plus urbains (les Bouches du Rhône - $398 \mathrm{hab} / \mathrm{km}^{2}$ ), avec des métropoles principalement côtières (Marseille, Toulon, Nice, Montpellier). 
Figure 1. Les régions méditerranéennes étudiées

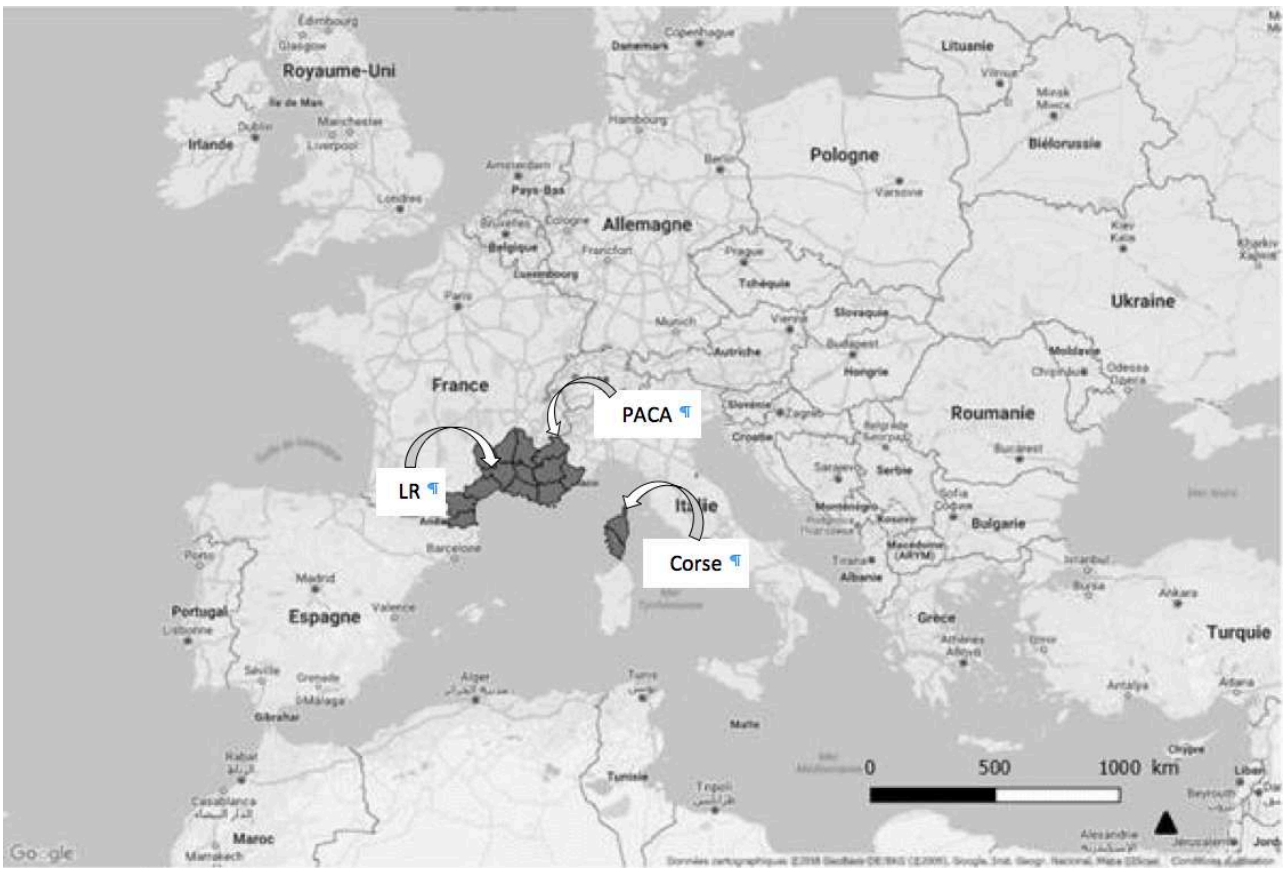

\section{Des données disponibles depuis 1973}

L'étude s'est appuyée sur des données recueillies à l'échelle municipale, sur une période de huit ans, de 2006 à 2013, issues de la base de données Prométhée ${ }^{43}$, qui recense tous les feux survenus dans la région depuis 1973. Elle fait une distinction entre les feux dits "Autres Feux de l'Espace Rural et Péri-Urbain » (AFERPU) et les feux de forêt, qui sont définis comme « les feux qui ont atteint et endommagé une zone de forêt ou d'arbustes couvrant plus de 1 ha, quelle que soit la zone brûlée ». Entre 2006 et 2013, 14057 feux ont été enregistrés sur la zone étudiée. Les données sur l'occupation des sols proviennent de la base de données Corine Land Cover (CLC) ${ }^{44}$, inventaire de l'occupation des sols qui distingue 44 classes et du Registre Parcellaire Graphique (RPG ${ }^{45}$ qui détaille les types de culture par zone. Les contraintes statistiques nous imposant de réduire le nombre de variables, nous avons regroupé les différentes classes en fonction des propositions de la littérature eu égard à leur effet sur le niveau de risque d'incendie. Ainsi, dans la base de données CLC, nous avons sélectionné trois types d'occupation des sols : les forêts, les zones arbustives et les terres agricoles hétérogènes (ayant un patron paysager complexe). Nous avons regroupé les données agricoles du RPG en six variables: cultures annuelles, vignobles, oliviers, arboriculture fruitière, pâturages extensifs et pâturages intensifs. Nous avons enfin identifié trois variables binaires indiquant la présence ou l'absence des interfaces suivantes : vignobles/forêts, céréales/ forêts et urbains/forêts. La reconnaissance des interfaces a été réalisée par le logiciel Qgis à partir de la mise à jour 2012 de la base de données CLC, tandis que les données sur l'usage du sol ont été générées à partir des mises à jour 2006 et 2012. Afin de prendre en compte d'autres caractéristiques spatiales et sociales susceptibles d'influer sur le départ ou l'intensité des feux de forêt, nous avons étendu notre analyse aux facteurs topographiques et biophysiques, mis à disposition par l'observatoire du Développement Rural ${ }^{46}$. Nous avons inclus l'altitude et la taille de la commune, ainsi 
que des variables tenant compte des contraintes climatiques telles que la vitesse moyenne mensuelle $d u$ vent, la température et les précipitations. Nous avons également utilisé deux variables indirectes pour le tourisme : le nombre de lits d'hôtel et de places de camping disponibles par rapport à la population de la municipalité, et le nombre de résidences secondaires par rapport au nombre total de résidences (Tableau 1).

Tableau 1. données utilisées

\begin{tabular}{|c|c|c|}
\hline Type de variable et source & Intitulé & Description \\
\hline \multirow{3}{*}{ Données communales, INSEE } & Altitude & Altitude moyenne de la commune \\
\hline & Superficie & $\begin{array}{l}\text { Superficie totale de la commune } \\
\text { (ha) }\end{array}$ \\
\hline & $\begin{array}{l}\text { Densité de } \\
\text { population }\end{array}$ & Population/surface \\
\hline \multirow{2}{*}{ Tourisme, INSEE $(2012,2013)$} & Tourism 1 & $\begin{array}{l}\text { Nombre de lits d'hôtels et de places } \\
\text { de camping en 2013/population }\end{array}$ \\
\hline & Tourism2 & $\begin{array}{l}\text { Nombre de résidences secondaires } \\
\text { en 2012/total des résidences }\end{array}$ \\
\hline \multirow{3}{*}{$\begin{array}{l}\text { Données climatiques, ODR - Observatoire Du } \\
\text { Développement Rural (annuelles) }\end{array}$} & Températures & $\begin{array}{l}\text { Température mensuelle moyenne } \\
\left({ }^{\circ} \mathrm{C}\right)\end{array}$ \\
\hline & Vents & $\begin{array}{l}\text { Vitesse mensuelle moyenne des } \\
\text { vents }(\mathrm{km} / \mathrm{h})\end{array}$ \\
\hline & Pluie & $\begin{array}{l}\text { Moyenne mensuelle des pluies } \\
(\mathrm{mm})\end{array}$ \\
\hline \multirow{6}{*}{$\begin{array}{l}\text { Usage du sol - CLC }(2006,2012) \text {. Données } \\
\text { annuelles à partir d'interpolation }\end{array}$} & Clc24_heterog & Zones agricoles hétérogènes (ha) \\
\hline & Clc31_forest & Forêts (ha) \\
\hline & Clc32_shrub & Friches (ha) \\
\hline & Int_for_cereal & $\begin{array}{l}\text { interface forêts-céréales } \\
(0=\text { absence } ; 1=\text { présence })\end{array}$ \\
\hline & Int_for_urban & $\begin{array}{l}\text { interface forêts-urbain ( } 0=\text { absence ; } \\
1=\text { présence) }\end{array}$ \\
\hline & $\begin{array}{l}\text { Int_for_ } \\
\text { vineyards }\end{array}$ & $\begin{array}{l}\text { interface forêts-vignes ( } 0=\text { absence ; } \\
1=\text { présence) }\end{array}$ \\
\hline \multirow{5}{*}{$\begin{array}{l}\text { Agriculture, Registre Parcellaire } \\
\text { Graphique (données annuelles entre } 2006 \\
\text { et 2013) }\end{array}$} & $\begin{array}{l}\text { Cultures } \\
\text { annuelles }\end{array}$ & $\begin{array}{l}\text { Céréales, oléo-protéagineux, } \\
\text { maraichages, fleurs (ha) }\end{array}$ \\
\hline & Vignes & \\
\hline & Oliviers & \\
\hline & Arboriculture & Vergers (ha) \\
\hline & $\begin{array}{l}\text { Pâturages } \\
\text { extensifs }\end{array}$ & Pâturages extensifs (ha) \\
\hline
\end{tabular}




\begin{tabular}{|l|l|}
$\begin{array}{l}\text { Pâturages } \\
\text { intensifs }\end{array}$ & $\begin{array}{l}\text { Fourrages, prairies permanentes et } \\
\text { temporaires (ha) }\end{array}$
\end{tabular}

$12 \mathrm{Au}$ total, nous disposons de 21704 observations concernant 2713 municipalités sur 8 ans; chaque observation porte sur une seule municipalité et sur un an. Si l'on considère le nombre d'incendies enregistrés annuellement par municipalité, 15816 municipalités en ont zéro, soit 72,87\% du total (tableau 2). En outre, 15,38\% d'entre elles n'ont enregistré qu'un seul incendie, tandis que les $11,75 \%$ restants en ont enregistré plus d'un.

Tableau 2. Nombre de feux enregistrés par année et par municipalité, entre 2006 et 2013

\begin{tabular}{|l|l|l|}
\hline Nombre de feux & Fréquence & Pourcentage \\
\hline 0 & 15816 & $72,87 \%$ \\
\hline 1 & 3338 & $15,38 \%$ \\
\hline$>1$ & 2550 & $11,75 \%$ \\
\hline
\end{tabular}

13 Entre 2006 et 2013, 12837 incendies ont été enregistrés dans la zone d'étude. 81 \% n'ont pas généré une surface brûlée supérieure à 1 ha. Moins nombreux, les grands incendies ont causé la majeure partie des dégâts (tableau 3).

Tableau 3. Distribution des feux par taille

\begin{tabular}{|l|l|l|}
\hline Étendue du feu & Fréquence & Pourcentage \\
\hline$>50 \mathrm{ha}$ & 110 & $0.86 \%$ \\
\hline$[10 \mathrm{ha}-50 \mathrm{ha}]$ & 336 & $2.62 \%$ \\
\hline$[1 \mathrm{ha}-10 \mathrm{ha}]$ & 1971 & $15.35 \%$ \\
\hline$[0,1 \mathrm{ha}-1 \mathrm{ha}]$ & 4040 & $31.47 \%$ \\
\hline$<0,1 \mathrm{ha}$ & 6380 & $49.70 \%$ \\
\hline Total & 12837 & $100.00 \%$ \\
\hline
\end{tabular}

14 Enfin, il est à noter que la variance annuelle de la superficie brulée indique des tendances pluriannuelles de croissance ou de régression des surfaces brulées (Figure 2). Sur notre période d'étude les deux tendances sont à l'œuvre. 
Figure 2. Total des superficies brulées par an (en ha)

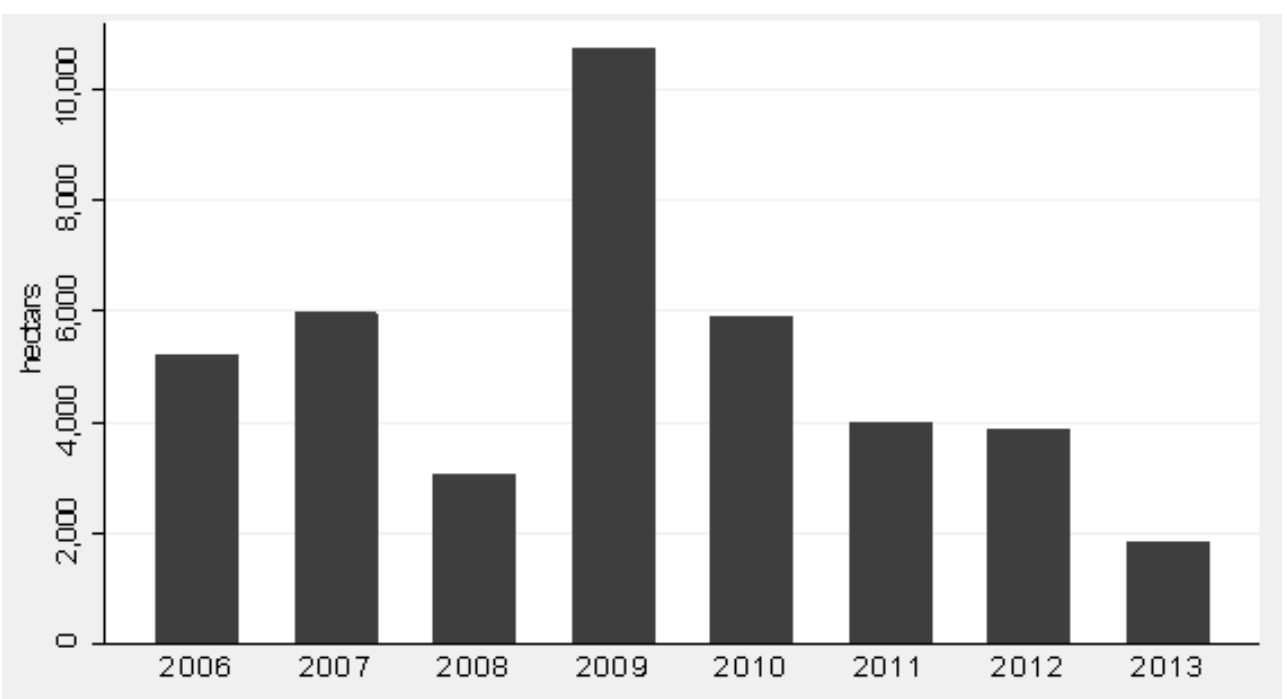

\section{Méthodologie}

Dans notre contexte, analyser l'effet de l'usage du sol sur le risque d'incendie nécessite de partir de deux types de risques : l'occurrence du feu (quelle que soit son amplitude ultérieure) et la propagation vers un incendie de grande ampleur. En effet, les deux phénomènes n'ont pas la même structure et leurs modélisations nécessitent des formes fonctionnelles différentes. Si le risque de propagation peut être analysé par une forme commune de type Logit qui donne des valeurs moyennes à partir d'une régression sur l'ensemble des données disponibles, l'occurrence des feux doit avoir un traitement plus sophistiqué parce qu'elle est caractérisée par un grand nombre de zéros issu de communes où il est impossible d'avoir un feu (par manque de forêt) concurremment à celles qui ont une structure paysagère à risque mais n'ont pas enregistré d'incendie l'année de l'observation.

La première étape a donc consisté à étudier l'impact de l'utilisation des terres agricoles sur le nombre d'occurrences d'incendies enregistré annuellement par municipalité. Les effets issus de données de comptage sont généralement estimés par des régressions de Poisson ou binomiales négatives mais, lorsqu'il y a une prépondérance de zéros, les modèles binomiaux dits à excès de zéros (que nous appellerons «Zinb" pour zeroinflated negative binomial) et à barrière (hurdle models) présentent plusieurs avantages. Le modèle dit à excès de zéros permet de croiser une distribution binaire (pour différencier les communes à 0 et les autres) avec une distribution ordinaire (pour le reste des variables) telle que Poisson ou binomiale négative ${ }^{47}$. Bien que similaire au modèle à barrière, il permet en outre d'analyser un certain nombre de zéros en même temps que les non-nuls. Dans ce cadre, les zéros observés peuvent être soit dits "d'échantillonnage ", c'est-à-dire le résultat du phénomène étudié, soit des zéros structurels dits "certains ", c'est-à-dire structurellement inévitables. L'intérêt du modèle à excès de zéros est donc qu'il existe deux processus distincts de génération de données nulles, l'un pour les zéros structurels et l'autre pour les autres résultats, y compris les zéros d'échantillonnage. Un test de Bernoulli permet alors de déterminer lequel des deux processus doit être employé. Une probabilité permet de déterminer si les zéros proviennent du premier ou du second processus ${ }^{48}$. La probabilité globale des 
zéros est la probabilité combinée de zéros des deux processus. Si le processus générant les zéros est $\mathrm{f}_{1}($.$) et le processus générant les réponses positives est \mathrm{f}_{2}($.$) alors le modèle$ est :

$$
g(y)= \begin{cases}f_{1}(0)+\left(1-f_{1}(0)\right) f_{2}(0) & \text { if } y=0 \\ \left.1-f_{1}(0)\right) f_{2}(y) & \text { if } y \geq 0\end{cases}
$$
par un seul feu était égale ou supérieure à 10 ha et de 0 lorsque la superficie était inférieure à 10 ha. Utiliser une variable catégorielle comme dépendante plutôt que de prendre directement la zone brûlée, permet de simplifier l'analyse sur les feux les plus importants, sachant que leur croissance ultérieure dépend principalement de l'efficacité de la lutte et des caractéristiques topographiques sur leurs trajectoires ${ }^{50}$. En ce qui concerne les covariables, nous avons inclus toutes les caractéristiques spatiales disponibles (détaillées dans la table 1). En outre, le modèle Logit intègre une variable muette pour le département, en postulant que les moyens de lutte contre les incendies diffèrent sensiblement selon le budget départemental. Il convient de noter que dans les deux régressions, le biais issu de l'hétérogénéité de la taille des municipalités a été contrôlé par un indicateur relatif (le pourcentage de la couverture terrestre par rapport à la superficie municipale totale).

\section{Résultats et discussion}

Les résultats des estimations statistiques, pour ce qui concerne les occurrences de feux (modèle Zinb), sont conformes à la littérature ou à l'intuition commune pour les variables climatiques et géographiques (table 4): les municipalités à forte densité de 
population et aux températures élevées en été plus touchées par les incendies de forêt, tandis que les pluies fréquentes diminuent la probabilité d'inflammation. Pour ce qui est du tourisme, les deux variables considérées produisent des effets opposés : le nombre de lits d'hôtel et de places de camping disponibles (tourism1) n'aggrave pas le risque d'incendie de forêt alors que le nombre de résidences secondaires (tourism2), qui correspond à une population supplémentaire, a le même effet que la densité non saisonnière de population et augmente la probabilité d'incendie.

21 En ce qui concerne l'effet de l'agriculture, seules les cultures annuelles et l'arboriculture diminuent le risque d'incendie, alors que les terres agricoles hétérogènes, les pâturages et les oliviers ont tendance à en augmenter la probabilité. Les cultures annuelles et l'arboriculture sont deux types de culture qui limitent la biomasse sur la parcelle, notamment herbagées sèche en été ; le signe négatif est donc intuitif. En revanche, l'effet positif des pâturages sur la probabilité d'incendie est plus contre-intuitif et met en lumière la situation particulière de la Corse où le brûlage des pâturages est une pratique usitée et qui a été identifiée comme augmentant le risque d'incendies incontrôlés ${ }^{51}$. Le résultat est plus complexe à interpréter pour les zones plantées d'oliviers, car il n'y a pas de raisons apparentes qui relient cette culture à un risque d'incendie plus élevé. Une explication possible est que les oliveraies sont plus souvent situées dans des endroits secs à proximité de zones arbustives, et donc à proximité de zones sujettes aux incendies. La partie inflatée du modèle Zinb correspond à la prédiction de la probabilité d'être un zéro certain, c'est-à-dire une municipalité ne pouvant connaitre aucun feu de forêt $\mathrm{du}$ fait de ses caractéristiques propres. Intuitivement, la couverture forestière et arbustive est un prédicteur négatif du zéro certain (c'est-à-dire positif des incendies), tout comme l'interface forêt-urbain, conformément aux résultats de Vasconcelos et al..$^{52}$ ou de Bajocco et Ricotta ${ }^{53}$. À l'inverse, l'interface vigne-forêt augmente la probabilité qu'une municipalité soit un zéro certain, mettant ici en lumière l'importance numérique des municipalités du Languedoc au sein desquelles la vigne occupe la quasi-totalité du territoire (avec quelques tâches forestières expliquant les interfaces non nulles).

Les résultats concernant l'étendue des incendies (la régression Logit) montrent ensuite que la vitesse du vent et les zones arbustives ont une influence positive sur la probabilité d'un grand incendie (intuitif), tandis que l'interface forêt-urbain a un effet préventif sur la propagation des incendies (focalisation des moyens de lutte dans les zones habitées). Par ailleurs, bien que les différents types de cultures, de pâturages et de prairies n'aient pas d'effet significatif sur la propagation des incendies, l'hétérogénéité des terres agricoles les minore. Il faut toutefois garder à l'esprit que la régression Logit a un faible pouvoir prédictif et nos résultats doivent donc être considérés avec prudence.

Il convient de mentionner les résultats opposés entre les deux modèles pour les variables rendant compte des interfaces forêts-urbain et des terres agricoles hétérogènes. Les interfaces forêts-urbain ont un effet négatif sur les zéros certains (les communes qui ne peuvent pas avoir de feux), c'est-à-dire qu'ils ont tendance à favoriser le nombre de départs de feux, alors qu'ils minorent leur extension. Cela signifie que l'anthropisation augmente les occurrences de feux mais contient leur propagation du fait des moyens qui y sont concentrés et de la valeur des biens à défendre; résultat conforme à l'étude de Catry et al. ${ }^{54}$, où les schémas spatiaux d'occurrence diffèrent en fonction de la taille du feu ; la majorité des grands incendies 
se produisant dans des territoires à densité de population relativement faible. Les terres agricoles hétérogènes minorent également la propagation des feux de grande ampleur. Elles correspondent aux espaces naturels entrecoupés d'usages agricoles extensifs et de territoires agro-forestiers, rendant compte de systèmes paysagers complexes au sein desquels les espaces agricoles ont un double effet de réduction de biomasse et de pourvoyeurs d'accès pour les services de lutte. Ces deux facteurs indiquent une probabilité plus élevée d'occurrences de feux d'origine humaine et, simultanément, des lieux où les pompiers peuvent intervenir plus rapidement pour prévenir la propagation des incendies, conformément aux résultats de Nunes et al. pour le Portugal ${ }^{55}$. La base de données Prométhée reste toutefois délicate à exploiter en ce sens qu'elle ne fait qu'une distinction entre les incendies forestiers et non forestiers. Les résultats de notre étude doivent être interprétés dans ce contexte : les différences par rapport aux autres études sont susceptibles d'être influencés par les différents types d'incendies analysés.

Table 4. Résultats de la régression 56

\begin{tabular}{|c|c|c|c|}
\hline \multicolumn{2}{|l|}{ ZINB } & \multicolumn{2}{|l|}{ LOGIT } \\
\hline Variable & $\begin{array}{l}\text { Significativité et signe } \\
\text { de la relation }\end{array}$ & Variable & $\begin{array}{l}\text { Significativité et signe } \\
\text { de la relation }\end{array}$ \\
\hline Altitude & & Altitude & \\
\hline $\begin{array}{l}\text { Superficie de la } \\
\text { commune }\end{array}$ & $+* * *$ & $\begin{array}{l}\text { Superficie de la } \\
\text { commune }\end{array}$ & \\
\hline Densité de population & $+* * *$ & Densité de population & \\
\hline $\begin{array}{l}\text { Température estivale } \\
\text { moyenne }\end{array}$ & $+* * *$ & $\begin{array}{l}\text { Température estivale } \\
\text { moyenne }\end{array}$ & \\
\hline Hygrométrie & $-* * *$ & $\begin{array}{l}\text { Vitesse mensuelle } \\
\text { moyenne du vent }\end{array}$ & $+* *$ \\
\hline $\begin{array}{l}\text { tourism1 (campings/ } \\
\text { hôtels) }\end{array}$ & $-*$ & $\begin{array}{l}\text { Moyenne des pluies } \\
\text { estivales }\end{array}$ & \\
\hline $\begin{array}{l}\text { tourism2 (résidences } \\
\text { secondaires) }\end{array}$ & $+* * *$ & $\begin{array}{l}\text { tourism1 (campings/ } \\
\text { hôtels) }\end{array}$ & \\
\hline $\begin{array}{l}\text { Agriculture } \\
\text { hétérogène }\end{array}$ & $+* * *$ & $\begin{array}{l}\text { tourism2 (résidences } \\
\text { secondaires) }\end{array}$ & \\
\hline Cultures annuelles & $-* * *$ & $\begin{array}{l}\text { Interfaces forêts/ } \\
\text { céréales }\end{array}$ & \\
\hline Vignes & & $\begin{array}{l}\text { Interfaces forêts/ } \\
\text { urbain }\end{array}$ & $-* * *$ \\
\hline Oliviers & $+* * *$ & $\begin{array}{l}\text { Interfaces forêts/ } \\
\text { vignes }\end{array}$ & \\
\hline Arboriculture & $-* *$ & Agriculture hétérogène & $-* * *$ \\
\hline Pâturages intensifs & $+* * *$ & Forêts & \\
\hline Pâturages extensifs & & Friches & $+* * *$ \\
\hline
\end{tabular}




\begin{tabular}{|l|l|l|l|}
\hline \multicolumn{2}{|l|}{ Contrôle des zéros certains } & Cultures annuelles & \\
\hline Forêts & $-* * *$ & Vignes & \\
\hline Friches forêts/ & $-* * *$ & Oliviers & \\
\hline $\begin{array}{l}\text { Interfaces } \\
\text { céréales }\end{array}$ & Arboriculture & \\
\hline $\begin{array}{l}\text { Interfaces forêts/ } \\
\text { urbain }\end{array}$ & $-* * *$ & Pâturages intensifs & \\
\hline $\begin{array}{l}\text { Interfaces forêts/ } \\
\text { vignes }\end{array}$ & $+* * *$ & Pâturages extensifs & \\
\hline$* * *$ p-value $<0,01-* * p-v a l u e<0,05-* p-v a l u e<0,1$ & \\
\hline
\end{tabular}

Enfin, un aspect considéré avec un intérêt croissant dans les analyses de l'évolution des schémas d'incendie de forêt, et que nous n'avons pas traité ici, est l'effet du changement de l'usage du sol lié aux activités de déforestation et à l'abandon des terres agricoles à proximité des zones naturelles ${ }^{57}$. Compte tenu de la courte période étudiée et de l'absence de référence spatiale précise pour les départs des feux de forêt, nous n'avons pas pu analyser l'effet de ces facteurs sur leur propagation. Ajouté au relativement faible pouvoir prédictif du modèle Logit sur la taille des incendies, l'incapacité à traiter cet aspect suggère qu'une analyse spatiale fine serait une extension utile de ce travail. L'examen de la localisation des terres agricoles par rapport aux zones brûlées devrait, par exemple, permettre de mieux comprendre la relation entre les zones brûlées et l'utilisation des terres agricoles. Enfin, notre étude a englobé des zones très diverses au sens de leur histoire et leur sociologie. La structure parcellaire héritée des usages anciens peut-être, par exemple, un facteur d'explication candidat de la relation entre départ et propagation : les incendies tendent à être plus difficilement maitrisable dans les espaces couverts d'une biomasse homogène, alors que les micro-parcellaires hérités, si tant est qu'ils correspondent toujours à un usage agricole, sont susceptibles de limiter la transformation de départs de feux en incendies de forêt. Par ailleurs, une approche plus fine pourrait appréhender la diversité des contextes sociaux locaux vis-à-vis de la pression incendiaire (en Corse versus Paca par exemple) et son lien avec l'agriculture (feux agricoles versus périurbains).

\section{ANNEXES}

Figure 7. Résultats du modèle Zinb 


\begin{tabular}{|c|c|c|c|c|c|c|}
\hline \multicolumn{3}{|c|}{ zero-inflated negative binomial regression } & \multicolumn{2}{|c|}{$\begin{array}{l}\text { Number of obs } \\
\text { Nonzero obs } \\
\text { Zero obs }\end{array}$} & $\begin{array}{l}= \\
= \\
=\end{array}$ & $\begin{array}{r}21,163 \\
5,738 \\
15,425\end{array}$ \\
\hline \multicolumn{2}{|c|}{$\begin{array}{l}\text { Inflation node1 }=\operatorname{logit} \\
\text { Log likelihood }=-18451.05\end{array}$} & \multicolumn{3}{|c|}{$\begin{array}{l}\text { LR chi2 } 2(21) \\
\text { Prob }>\text { chi2 }\end{array}$} & \multicolumn{2}{|r|}{$\begin{array}{r}3575.83 \\
0.0000\end{array}$} \\
\hline num_fires & coef. & std. Err. & $z$ & $P \&|z|$ & [95\% conf. & f. Interval] \\
\hline \multicolumn{7}{|l|}{ num_fires } \\
\hline altitude & -.0000881 & .000093 & -0.95 & 0.343 & -.0002704 & .0000942 \\
\hline surface & .0001633 & $5.87 e-06$ & 27.80 & 0.000 & .0001518 & .0001748 \\
\hline pop_density & .0320372 & .00517 & 6.20 & 0.000 & .0219042 & .0421702 \\
\hline temp & .1557897 & .015735 & 9.90 & 0.000 & .1249497 & .1866298 \\
\hline rain & -0191361 & .0010549 & -18.14 & 0.000 & -0212037 & -0170685 \\
\hline tour isne1 1 & -.0440886 & .0248551 & -1.77 & 0.076 & -.0928036 & .0046265 \\
\hline tour isne2 2 & .3179573 & .0855246 & 3.72 & 0.000 & .1503323 & .4855824 \\
\hline c1c24_heterog & .4511681 & .1558824 & 2.89 & 0.004 & .1456441 & .756692 \\
\hline annual_crops & -4.547742 & .335609 & $-13,55$ & 0.000 & -5.205524 & -3.88996 \\
\hline vineyards & .2525699 & .2862048 & 0.88 & 0.378 & -.3083811 & .8135209 \\
\hline olive_trees & 9.222166 & 2.654985 & 3.47 & 0.001 & 4,018491 & 14.42584 \\
\hline arboricultre & -2.836594 & 1.226187 & -2.31 & 0.021 & -5.239875 & $\therefore 4333117$ \\
\hline inten_past & 1.116855 & .273078 & 4.09 & 0.000 & .5816321 & 1.652078 \\
\hline exten_past & .0528105 & .1317738 & 0.46 & 0.689 & $\therefore 2054614$ & .3110824 \\
\hline \multicolumn{7}{|l|}{ year } \\
\hline 2007 & .1666687 & .0557538 & 2.98 & 0.003 & .0567932 & .2753442 \\
\hline 2008 & $\therefore 2389494$ & .0582644 & -4.10 & 0.000 & $\begin{array}{r}-3531456 \\
\end{array}$ & $\begin{array}{r}-1247533 \\
-12438\end{array}$ \\
\hline 2009 & -.2072481 & .0537392 & -3.86 & 0.000 & -.312575 & -1019211 \\
\hline 2010 & $\begin{array}{r}-1642758 \\
\end{array}$ & .0580743 & $\begin{array}{l}-2.83 \\
\end{array}$ & 0.005 & $\begin{array}{r}-2780994 \\
-.2789\end{array}$ & $\begin{array}{r}\therefore 0504523 \\
-\therefore\end{array}$ \\
\hline 2011 & .3913639 & .0594853 & 6.58 & 0.000 & $\begin{array}{r}.2747749 \\
\end{array}$ & .5079529 \\
\hline 2012 & $\therefore 2052155$ & .0546683 & -3.75 & 0.000 & $\begin{array}{r}-3123635 \\
-.3129\end{array}$ & $\begin{array}{r}\therefore 0980676 \\
-.09679\end{array}$ \\
\hline 2013 & $\therefore 2542892$ & .0598848 & -4.25 & 0.000 & -.3716613 &. .1369171 \\
\hline _cons & -3.485625 & .3994169 & -8.73 & 0.000 & -4.268467 & -2.702782 \\
\hline \multicolumn{7}{|l|}{ inflate } \\
\hline clc31_forest & $\therefore 0010016$ & .0001658 & -6.04 & 0.000 & -.0013266 & $\therefore .0006766$ \\
\hline C32_shrub & -.0048485 & .0004247 & -11.42 & 0.000 & -.0056809 & -.0040161 \\
\hline int_for_cereal & $\therefore 2082619$ & .154193 & -1.35 & 0.17 & $\therefore .5104745$ & .0939508 \\
\hline int_for_urban & $\therefore 8098708$ & .1228479 & -6.59 & 0.000 & -1.050648 & $\begin{array}{r}.5699933 \\
\end{array}$ \\
\hline int_for_vineya-s & .5283218 & .1170279 & 4.51 & 0.000 & .2989513 & .7576924 \\
\hline cons & .3886374 & .0988766 & 3.93 & 0.000 & .1948429 & .5824319 \\
\hline /1nalpha & .2219152 & .035317 & 6.28 & 0.000 & .1526951 & .2911353 \\
\hline alpha & 1.248466 & .0440921 & & & 1.16497 & 1.337946 \\
\hline
\end{tabular}

Likelihood-ratio test of alpha=日: chibar2(01) $=4187,01$ Prs=chibar2 $=0.0000$ warning: The vuong test is not appropriate for testing zero-inflation.

Figure 8. Résultats de la régression Logit

Image 1004AE6C0000492F0000593310D2A1858B8A8FD2.emf

\begin{tabular}{|c|c|}
\hline ogistic regression & $\begin{array}{l}\text { Number of obs } \\
\text { LR chi2(32) } \\
\text { Prob }>\text { chi2 }\end{array}$ \\
\hline & Pseudo R2 \\
\hline
\end{tabular}

\begin{tabular}{|c|c|c|c|c|c|c|}
\hline over10 & coef. & Std. Err. & $z$ & $P>|z|$ & [95\% conf. & Interval] \\
\hline temp & .0135639 & .0086587 & 1.57 & $\theta .117$ & -.0034069 & .0305347 \\
\hline wind & .1107416 & .0554395 & 2.00 & $\theta .046$ & $.002 \theta 821$ & .219401 \\
\hline rain & .0005275 & .0008867 & $\theta .59$ & 0.552 & -.0012104 & .0022654 \\
\hline altitude & .0001307 & $.00 \Theta 2 \theta 76$ & $\theta .63$ & $\theta .529$ & -.0002762 & .0005377 \\
\hline surface & .0000169 & .0000136 & 1.24 & 0.213 & $-9.74 e-06$ & .0000436 \\
\hline pop_density & -.0207232 & .0172184 & $-1.2 \theta$ & 0.229 & -.0544706 & .0130241 \\
\hline tour isme 1 & - . 0191402 & .0843849 & -0.23 & 0.821 & -.1845315 & .1462512 \\
\hline tourisme 2 & .0929329 & .3171896 & $\theta .29$ & $\theta .77 \theta$ & -.5287473 & .7146132 \\
\hline int_for_cereal & -.2007527 & .1688806 & -1.19 & 0.235 & -.5317527 & .1302472 \\
\hline int_for_urban & -.320333 & .1217179 & -2.63 & $\theta .008$ & -.5588957 & -.0817704 \\
\hline t_for_vineya-s & .035503 & .1299818 & 0.27 & 0.785 & -.2192566 & .2902626 \\
\hline clc24_heter & -1.733425 & .6602411 & -2.63 & $\theta .009$ & -3.027474 & -.4393764 \\
\hline clc31_forest & -.1518656 & .3684034 & -0.41 & 0.680 & -.873923 & .5701918 \\
\hline clc32_shrub & 1.130524 & .3904257 & 2.90 & 0.004 & .3653037 & 1.895744 \\
\hline annual_crops & 1.538171 & 1.268993 & 1.21 & 0.225 & -.9490091 & 4. 02535 \\
\hline vineyards & -.5432413 & 1.083833 & -0.50 & 0.616 & -2.667516 & 1.581033 \\
\hline olive_trees & 3.089463 & 9.796144 & 0.32 & 0.752 & -16.11063 & 22.28955 \\
\hline arboricultre & 5.337256 & 4.689412 & 1.14 & 0.255 & -3.853822 & 14.52833 \\
\hline inten_past & -1.286027 & 1.232771 & -1.04 & 0.297 & -3.702214 & 1.130159 \\
\hline exten_past & .6639956 & .4722536 & 1.41 & $\theta .160$ & -.2616045 & 1.589596 \\
\hline dep_co & & & & & & \\
\hline haute-alpes & .2625386 & .5877992 & $\theta .45$ & 0.655 & -.8895266 & 1.414604 \\
\hline pes maritimes & -.4612039 & .303133 & -1.52 & 0.128 & -1.055334 & .1329259 \\
\hline aude & -.9573025 & .3462457 & -2.76 & $\theta .006$ & -1.635932 & -.2786733 \\
\hline uches-du-rh-e & -1.045308 & .3469192 & -3.01 & $\theta .003$ & -1.725257 & -.3653585 \\
\hline corse du sud & -1.723494 & .3206109 & -5.38 & $\theta . \theta 0 \theta$ & -2.35188 & -1.095109 \\
\hline haute-corse & -1.226579 & .2983364 & -4.11 & $\theta .00 \theta$ & -1.811307 & -.6418503 \\
\hline gard & -.2793559 & .3570811 & -0.78 & 0.434 & -.979222 & .4205102 \\
\hline herault & -.2352299 & .3071999 & $-\theta .77$ & 0.444 & -.8373305 & .3668708 \\
\hline lozere & -.2029914 & .3281383 & -0.62 & $\theta .536$ & -.8461306 & .4401478 \\
\hline pyrenees orie.. & -.0311923 & .3246441 & -0.10 & 0.923 & -.667483 & .6050984 \\
\hline var & -.8586482 & .3263995 & -2.63 & 0.009 & -1.498379 & -.218917 \\
\hline vaucluse & -.5193313 & .4700181 & $-1.1 \theta$ & 0.269 & -1.44055 & .4018871 \\
\hline _cons & -3.181625 & .4917394 & 47 & $\theta . \theta 0 \theta$ & -4.145417 & -2.217834 \\
\hline
\end{tabular}




\section{NOTES}

1. Marco Turco, Joaquín Bedia, Fabrizio Di Liberto et al., «Decreasing Fires in Mediterranean Europe », PLoS One, vol. 11, n 3, 2016, DOI : 10.1371/journal.pone.0150663.

2. Marco Turco, Maria-Carmen Llasat, Jost Hardenberg et Antonello Provenzale, «Climate change impacts on wildfires in a Mediterranean environment ", Climatic Change, $\mathrm{n}^{\circ} 125,2014$, p. 369-380. DOI : 10.1007/s10584-014-1183-3.

3. Laura A. Burkle, Jonathan A. Myers et Travis Belote, « Wildfire disturbance and productivity as drivers of plant species diversity across spatial scales ", Ecosphere, $\mathrm{n}^{\circ} 6,2015$, DOI : 10.1890/ ES15-00438.1.

4. Patricia M. Alexandre, Miranda H. Mockrin, Susan I. Stewart et al., "Rebuilding and new housing development after wildfire ", International Journal of Wildland Fire, $\mathrm{n}^{\circ} 24,2015$, p. 138-149. DOI : 10.1071/WF13197.

5. George P. Petropoulos, Hywel M. Griffiths et Dionissios P. Kalivas, « Quantifying spatial and temporal vegetation recovery dynamics following a wildfire event in a Mediterranean landscape using EO data and GIS », Applied Geography, n 50, 2014, p. 120-131.

6. Daniel Alexandrian et Maurice Gouiran, "Les causes d'incendie: levons le voile ", Revue forestière française, vol. 42, 1990, en ligne : http://documents.irevues.inist.fr/bitstream/handle/ 2042/26158/RFF_1990_S_33.pd ?sequence=1.

7. Francisco Moreira, Olga Viedma, Margarita Arianoutsou et al., «Landscape-wildfire interactions in southern Europe: implications for landscape management", Journal of environmental management, vol. 92, $\mathrm{n}^{\circ}$ 10, 2011, p. 2389-2402.

8. San-Miguel-Ayanz, J. et al., Feux de forêt en Europe, au Moyen-Orient et en Afrique du Nord 2014, Direction générale de l'environnement (Commission européenne), Institut pour l'environnement et la durabilité (Centre commun de recherche), 2015, DOI (pdf) : 10.2788/224527.

9. Juli G. Pausas, "Changes in fire and climate in the eastern Iberian Peninsula (Mediterranean basin) ", Climatic change, $\mathrm{n}^{\circ}$ 6, 2004, p. 337-350 ; Maria C.S. Nunes, Maria J. Vasconcelos, José M.C. Pereira et al., "Land cover type and fire in Portugal : do fires burn land cover selectively?", Landscape Ecology, $\mathrm{n}^{\circ}$ 20, 2005, p. 661-673 ; Luis Galiana-Martín, « The wildland-urban interface : a risk prone area in Spain ", Proceedings of the 5th International Wildland Fire Conference, Sun City, 2011, www.wildfire2011.org/material/papers/Luis_Galiana-Martin.pdf ; José M. Moreno, Olga Viedma, Gonzalo Zavala et Belén Luna, «Landscape variables influencing forest fires in central Spain », International Journal of Wildland Fire, $\mathrm{n}^{\circ} 20,2011$, p. 678-689 ; Sofia L. J. Oliveira, José M.C. Pereira et Joao Carreiras, «Fire frequency analysis in Portugal (1975-2005), using Landsat-based burnt area maps », International Journal of Wildland Fire, $n^{\circ} 21,2012$, p. 48-60.

10. European Commission, Joint Research Centre, Institute for Environment and Sustainability, Forest fires in Europe 2010, report $n^{\circ} 11,2011$, Publications Office, Luxembourg.

11. Romain Louvet, Didier Josselin, Cyrille Genre-Granpierre et Jagannath Aryal, «Impact du changement d'échelle sur l'étude des causes des feux de forêts du sud-est de la France », 2016, en ligne : https://hal.archives-ouvertes.fr/hal-01249882.

12. Sofia Bajocco et Carlo Ricotta, «Evidence of selective burning in Sardinia (Italy) : which landcover classes do wildfires prefer? ", Landscape Ecology, $\mathrm{n}^{\circ}$ 23, 2008, p. 241-248.

13. N. Stamou, A. Christodoulou, V. Blioumis et S. Fotiou, "Effectiveness and efficiency of improvements of the forest fine protection system in Greek forests ", MEDIT, n ${ }^{\circ} 4 / 97,1997$, p. 37-44.

14. Josep Piñol, Jaume Terradas et Francisco Lloret, «Climate warming, wildfire hazard, and wildfire occurrence in coastal eastern Spain », Climatic change, vol. 38, n 3, 1998, p. 345-357, DOI : 10.1023/A:1005316632105.

15. Ricardo Díaz-Delgado, Francisco Lloret et Xavier Pons, «Statistical analysis of fire frequency models for Catalonia (NE Spain, 1975-1998) based on fire scar maps from Landsat MSS data ", 
International Journal of Wildland Fire, $\mathrm{n}^{\circ} 13,2004$, p. 89-99 ; Filipe X. Catry, Francisco Rego, Joaquim S. Silva et al., «Fire starts and human activities », Towards Integrated Fire Management-Outcomes of the European Project fire Paradox, 2010, p. 21-35; Anne Ganteaume et Marielle Jappiot, «What causes large fires in Southern France ", Forest Ecology and Management, $n^{\circ} 294,2013$, p. 76-85, DOI : 10.1016/j.foreco.2012.06.055.

16. Juli G. Pausas, « Changes in fire and climate in the eastern Iberian Peninsula... », art. cit.

17. Ricardo Díaz-Delgado, Francisco Lloret et Xavier Pons, «Statistical analysis of fire frequency models ", art. cit. ; Pierre Carrega, «Le risque d'incendies de forêt en région méditerranéenne : compréhension et évolution », 2008, en ligne : https://hal.archives-ouvertes.fr/hal-00470225.

18. Jeffrey P. Prestemon, John M. Pye, David T. Butry et al., « Understanding broadscale wildfire risks in a human-dominated landscape », Forest science, $\mathrm{n}^{\circ} 48,2002$, p. 685-693 ; Van Butsic, Maggi Kelly et Max A. Moritz, "Land use and wildfire: a review of local interactions and teleconnections", Land, $\mathrm{n}^{\circ}$ 4, 2015, p. 140-156; Olga Viedma, José M. Moreno, Cumhur Güngöroglu et al., « Recent land-use and land-cover changes and its driving factors in a fire-prone area of southwestern Turkey ", Journal of environmental management, $\mathrm{n}^{\circ} 197,2017, \mathrm{p} .719-731$, DOI : 10.1016/j.jenvman.2017.02.074.

19. Anne Ganteaume, Andrea Camia, Marielle Jappiot et al., « A review of the main driving factors of forest fire ignition over Europe ", Environmental management, $\mathrm{n}^{\circ}$ 51, 2013, p. 651-662.

20. Florent Mouillot, Jean-Pierre Ratte, Richard Joffre et al., « Some determinants of the spatiotemporal fire cycle in a Mediterranean landscape (Corsica, France) », Landscape Ecology, ${ }^{\circ} 18$, 2003, p. 665-674; Sofia Bajocco et Carlo Ricotta, « Evidence of selective burning », art. cit.

21. Filipe X. Catry, Francisco Rego, Joaquim S. Silva et al., « Fire starts and human activities », art. cit. ; Pierre Carrega, «Le risque d'incendies de forêt », art. cit. ; Corinne Lampin, Marielle Jappiot et Jean-Paul Ferrier, " Modélisation du risque d'incendie de forêt dans les interfaces habitatsforêts ", Sciences Eaux et Territoires, 2011, DOI : 10.14758/SET-REVUE.2011.HS.03 ; Anne Ganteaume et Marielle Jappiot, « What causes large fires », art. cit.

22. Maria José Perestrello de Vasconcelos, Sara Silva, Margarida Tomé et al., «Spatial prediction of fire ignition probabilities: comparing logistic regression and neural networks", Photogrammetric engineering and remote sensing, $\mathrm{n}^{\circ}$ 67, 2001, p. 73-81; Monica Mermoz, Thomas Kitzberger et Thomas T. Veblen, «Landscape influences on occurrence and spread of wildfires in Patagonian forests and shrublands », Ecology, n 86, 2005, p. 270-271; Francisco Moreira, Olga Viedma, Margarita Arianoutsou et al., « Landscape-wildfire interactions », art. cit.

23. José M. Moreno, Olga Viedma, Gonzalo Zavala et Belén Luna, «Landscape variables influencing forest fires ", art. cit.

24. Anne Ganteaume et Marielle Jappiot, « What causes large fires », art. cit.

25. L.R. Donoghue et W.A. Main, «Some factors influencing wildfire occurrence and measurement of fire prevention effectiveness ", Journal of Environmental Management, $n^{\circ} 20,1985$, p. 87-96; Daniel E. Mercer et Jeffrey P. Prestemon, « Comparing production function models for wildfire risk analysis in the wildland-urban interface ", Forest policy and economics, vol. 7, n ${ }^{\circ} 5$, 2005, p. 782-795.

26. Olga Viedma, José M. Moreno, Cumhur Güngöroglu et al., « Recent land-use and land-cover changes ", art. cit.

27. Maria José Perestrello de Vasconcelos, Sara Silva, Margarida Tomé et al., "Spatial prediction ", art. cit. ; Sofia Bajocco et Carlo Ricotta, « Evidence of selective burning ", art. cit.

28. Maria José Perestrello de Vasconcelos, Sara Silva, Margarida Tomé et al., "Spatial prediction », art. cit. ; Olga Viedma, José M. Moreno, Cumhur Güngöroglu et al., « Recent land-use and land-cover changes ", art. cit.

29. Daniel E. Mercer et Jeffrey P. Prestemon, « Comparing production function models », art. cit.

30. Matt P. Plucinski, A review of wildfire occurrence research, Australia, Bushfire Cooperative Research Centre, 2011 (pdf). 
31. David L. Martell, Sam Otukol et Brian J. Stocks, « A logistic model for predicting daily peoplecaused forest fire occurrence in Ontario ", Canadian Journal of Forest Research, n ${ }^{\circ} 17,1987$, p. 394-401, https//doi.org/10.1139/x87-068; Cristina Vega-Garcia et al., «A logit model for predicting the daily occurrence of human caused forest-fires ", International Journal of Wildland Fire, $n^{\circ} 5,1995$, p. 101-111 ; B.D. Amiro et al., «Fire weather index system components for large fires in the Canadian boreal forest ", International Journal of Wildland Fire, $n^{\circ} 1,2005$, p. 391-400.

32. Maria C.S. Nunes, Maria J. Vasconcelos, José M.C. Pereira et al., «Land cover type ", art. cit.; Francisco Moreira, Pedro Vaz, Filipe Xavier Catry et Joaquim Sande Silva, « Regional variations in wildfire susceptibility of land-cover types in Portugal : implications for landscape management to minimize fire hazard ", International Journal of Wildland Fire, vol. 18, $n^{\circ} 5,2009$, p. 563-574, DOI : https://doi.org/10.1071/WF07098; Filipe X. Catry, Francisco Rego, Joaquim S. Silva et al., "Fire starts and human activities », art. cit. ; Olga Viedma, José M. Moreno, Cumhur Güngöroglu et al., « Recent land-use and land-cover changes", art. cit.

33. Monica Mermoz, Thomas Kitzberger et Thomas T. Veblen, «Landscape influences », art. cit.; Wisdom Dlamini, «A Bayesian belief network analysis of factors influencing wildfire occurrence in Swaziland ", Environmental Modelling \& Software, $n^{\circ} 25,2010$, p. 199-208.

34. Jeffrey P. Prestemon, John M. Pye, David T. Butry et al., « Understanding broadscale wildfire risks ", art. cit.

35. Ricardo Díaz-Delgado, Francisco Lloret et Xavier Pons, "Statistical analysis of fire frequency models », art. cit. ; Francisco Moreira, Pedro Vaz, Filipe Xavier Catry et Joaquim Sande Silva, « Regional variations ", art. cit.

36. Daniel E. Mercer et Jeffrey P. Prestemon, « Comparing production function models », art. cit. 37. David L. Martell, Sam Otukol et Brian J. Stocks, « A logistic model », art. cit.

38. Maria José Perestrello de Vasconcelos, Sara Silva, Margarida Tomé et al., «Spatial prediction", art. cit.; Filipe X. Catry, Francisco Rego, Joaquim S. Silva et al., "Fire starts and human activities ", art. cit.

39. D. Mandallaz et R. Ye, "Prediction of forest fires with Poisson models », Canadian Journal of Forest Research, $\mathrm{n}^{\circ} 27,1997$, p. 1685-1694.

40. D.X. Viegas, M.T.S.P. Viegas et A.D. Ferreira, " Moisture content of fine forest fuels and fire occurrence in central Portugal ", International Journal of Wildland Fire, vol. 2, n² 2, 1992, p. 69-86; Jeffrey P. Prestemon, John M. Pye, David T. Butry et al., « Understanding broadscale wildfire risks ", art. cit.

41. Daniel E. Mercer et Jeffrey P. Prestemon, " Comparing production function models ", art. cit.

42. Les données dont nous disposons datant d'avant 2015, nous retenons l'ancienne formulation et géographie de la région, sachant qu'elle est devenue depuis l'Occitanie.

43. Voir www.promethee.com.

44. Corine Land Cover est un inventaire de l'usage du sol à l'échelle européenne - voir https:// www.statistiques.developpement-durable.gouv.fr/corine-land-cover-0.

45. Le recensement parcellaire graphique est l'inventaire des parcelles des exploitations françaises ayant reçu une prime PAC - voir https://www.data.gouv.fr/fr/datasets/.

46. https://odr.inra.fr/intranet/carto_joomla/

47. Charles E. Rose, Stacey W. Martin, Kathleen A. Wannemuehler et B.D. Plikaytis, « On the Use of Zero-Inflated and Hurdle Models for Modeling Vaccine Adverse Event Count Data ", Journal of Biopharmaceutical Statistics, vol. 16, n 4, 2006, p. 463-481, DOI : 10.1080/10543400600719384.

48. O. Yusuf, T. Bello et O. Gureje, «Zero Inflated Poisson and Zero Inflated Negative Binomial Models with Application to Number of Falls in the Elderly », Biostatistics and Biometrics Open Access Journal, Juniper Publishers Inc., vol. 1, 2017, p. 69-75, DOI : 10.19080/BBOAJ.2017.01.555566.

49. Plan de Protection des Forêts et des Espaces Naturels contre les Incendies en Corse, Cahier 2, Documents techniques et graphiques, 2013. 
50. Éléments issus des dires d'experts (pompiers et responsables de luttes contre les incendies), sollicités lors de notre travail.

51. Florent Mouillot, Jean-Pierre Ratte, Richard Joffre et al., « Some determinants », art. cit. ; Plan de Protection des Forêts et des Espaces Naturels contre les Incendies en Corse, Cahier 1, Document d'orientation et fiches actions.

52. Maria José Perestrello de Vasconcelos, Sara Silva, Margarida Tomé et al., "Spatial prediction », art. cit.

53. Sofia Bajocco et Carlo Ricotta, « Evidence of selective burning ", art. cit.

54. Filipe Xavier Catry, Francisco Rego, Fernando Baçao et Francisco Moreira, « Modeling and mapping wildfire ignition risk in Portugal », International Journal of Wildland Fire, vol. 18, $\mathrm{n}^{\circ} 8$, 2010, p. 921-931.

55. Maria C.S. Nunes, Maria J. Vasconcelos, José M.C. Pereira et al., « Land cover type », art. cit.

56. Les variables du modèle à excès de zéros permettent de prédire la probabilité d'avoir un zéro certain, c'est-à-dire une commune ne pouvant pas connaitre de feux de forêt; le signe du coefficient +/- représentent le sens de la relation et $* / * * / * * *$ le niveau de significativité (suffisante mais faible/moyenne/très bonne - l'absence d'information correspond à une non significativité de la variable).

57. Francisco Moreira, Pedro Vaz, Filipe Xavier Catry et Joaquim Sande Silva, «Regional variations », art. cit. ; Olga Viedma, José M. Moreno, Cumhur Güngöroglu et al., « Recent land-use and land-cover changes », art. cit.

\section{RÉSUMÉS}

La région méditerranéenne est sujette aux incendies de forêt alors que les préoccupations écologiques génèrent un engagement institutionnel important dans la lutte. Toutefois, la question de la prévention reste ouverte : s'il est convenu que la majeure part des incendies de forêt est causée par l'homme, il n'existe pas un modèle consensuel permettant de localiser cette relation. Une voie est d'estimer le rôle que joue l'utilisation des terres dans le nombre ou l'intensité des incendies passés. Dans cette perspective, nous examinons l'impact de l'agriculture sur les incendies via une analyse quantitative de l'effet de l'usage agricole des terres sur leur occurrence et leur étendue. Nos résultats permettent de dresser le profil des municipalités qui sont plus exposées au risque d'incendie de forêt eu égard à la présence de certains types de cultures ou d'activités agricoles. Ils témoignent toutefois d'une relation complexe pour ce qui est de l'espace agricole dans son ensemble, qui peut être caractérisée à partir d'une classification raisonnée des productions (le maraichage minore les occurrences et les parcours extensifs les accroissent) ou des dynamiques spatiales (les espaces abandonnés minorent les occurrences et majorent les étendues).

The Mediterranean region is prone to wildfires while ecological concerns generate a strong institutional commitment to the fight. However, the question of prevention remains open: while it is agreed that the majority of wildfires are human-caused, there is no consensus model to localize this relationship. One way is to estimate the role that land use plays in the number or intensity of past fires. To this end, we examine the impact of agriculture on fire via a quantitative analysis of the effect of agricultural land use on fire occurrence and extent. Our results provide a profile of municipalities that are more exposed to wildfire risk due to the presence of certain 
types of crops or agricultural activities. However, they show a complex relationship with respect to the agricultural space as a whole, which can be characterized from a reasoned classification of productions (market gardening reduces occurrences and extensive rangelands increase them) or spatial dynamics (abandoned spaces reduce occurrences and increase extents).

\section{INDEX}

Mots-clés : zone méditerranéenne, Incendies de forêt, zones agricoles, déterminants des départs de feux

Keywords : Mediterranean area, forest fires, agricultural areas, fire ignition drivers

\section{AUTEURS}

FRANCESCO GUERRA

Francesco Guerra est économètre (INRAE-SMART LERECO, Site Agrocampus, Rennes). Il a réalisé une étude sur la relation entre les incendies de forêts et l'agriculture à l'Inrae de Corse. Il est maintenant doctorant à l'Inrae et travaille sur les cadres théoriques s'inscrivant dans la lignée des travaux récents de l'économie internationale basés sur les stratégies des firmes.

\section{CLAUDE NAPOLÉONE}

Claude Napoléone est économiste de l'environnement et des ressources naturelles (INRAE, Unité Écodéveloppement, Site Agroparc, Avignon). Il développe des modèles d'économie urbaine et d'économie régionale destinés à représenter les dynamiques d'usages des sols et leurs déterminants, principalement à partir d'approches pluridisciplinaires avec des géographes (géomaticiens), des agronomes et des écologues. Ses terrains d'étude sont localisés dans la zone méditerranéenne et les espaces de montagne.

\section{JEAN CHRISTOPHE PAOLI}

Jean Christophe Paoli est agro-économiste (INRAE-LRDE, Corte). Spécialiste des structures agraires des zones pastorales, il mobilise dans ses recherches les méthodes et concepts de l'agriculture comparée. Il s'intéresse en particulier aux relations foncières entre éleveurs et propriétaires et à l'évolution de l'occupation de l'espace montagnard. Il est responsable de l'implantation de Corte (LRDE) de l'UMR Systèmes d'élevage Méditerranéens et Tropicaux (SELMET). Ses terrains d'étude, outre la Corse sont situés en Italie (Sardaigne) et en Afrique du Nord (Tunisie), deux pays où il a séjourné.

\section{MICHEL MOULERY}

Michel Mouléry, spécialisé en géographie quantitative à l'INRAE d'Avignon depuis 2009 (Unité Écodéveloppement). Il s'intéresse sur les sujets mettant en relation l'agriculture ou le naturel souvent associé aux thématiques du territoire : étude des relations fonctionnelles ville/ agriculture, déterminants des changements de l'occupation du sol en focus l'agriculture, durabilité des prairies permanentes, impact de l'agriculture sur l'éclosion et la propagation des feux de forêt... Pour plus d'informations : https://www6.paca.inrae.fr/ecodeveloppement/LesFemmes-et-Les-Hommes/Moulery-Michel. 